Abstract 0C-126 Table 1 Results

\begin{tabular}{|c|c|c|c|c|c|}
\hline Outcome & VMMC (1) & Prepathway (2) & Nonpathway (3) & Pathway (4) & p Value \\
\hline Pts 1st day mobilisation (\%) & 93 & 8.3 & 42 & 100 & $\begin{array}{l}>0.99(1 \text { vs } 4) \\
<0.05 \text { (2 vs } 4) \\
0.16 \text { (2 vs } 3)\end{array}$ \\
\hline Complications (\%) & 47.3 & 75 & 75 & 33.3 & $\begin{array}{l}0.53 \text { (1 vs } 4) \\
<0.05 \text { (2 vs } 4)\end{array}$ \\
\hline ICU stay (days) & $1(0-22)$ & $4(2-20)$ & $3(2-9)$ & $3(1-5)$ & $\begin{array}{l}<0.05 \text { ( } 1 \text { vs } 4,2 \text { vs } 4) \\
0.13 \text { ( } 2 \text { vs } 3)\end{array}$ \\
\hline Hospital stay (days) & $8(6-54)$ & $17(12-30)$ & $13(8-22)$ & $7(6-37)$ & $\begin{array}{l}0.25 \text { (1 vs } 4) \\
<0.05 \text { (2 vs } 4) \\
0.09 \text { (2 vs } 3 \text { ) }\end{array}$ \\
\hline
\end{tabular}

Methods Four groups were included in this study:

1. 74 patients operated on at VMMC (2009-2011).

2. 12 RSC patients operated on before the introduction of SPCP.

3. 12 RSC patients operated on after the introduction of SPCP but not included.

4. 12 RSC patients managed according to the SPCP.

Groups were compared with respect to patient demographics, peri- and post-operative outcomes.

Results Presenting demographics were similar in all groups with respect to BMI, medical co-morbidities, ASA grade and clinical stage. Age was significantly reduced Group 4 compared to Group 2 $(\mathrm{p}<0.05)$. Following initiating SPCP the use of feeding jejunostomies increased $(67 \%-100 \% ; \mathrm{p}<0.05)$ as did immediate extubation $(67 \%-$ $100 \% ; p<0.05)$. Further statistically significant improvements were noted in complications, length of ICU and hospital stay. Group 3 also showed a less significant improvement in length of ICU and hospital stay, and first day mobilisation.

Conclusion The results of this study demonstrate the potential for accelerated improvement in short-term outcomes following oesophagectomy through the translocation and integration of standardised postoperative pathways. SPCP can also change hospital processes to improve outcomes in other patients. A multidisciplinary approach with involvement of the entire health care team in the design, implementation, evaluation and revision of these pathways is essential and will help ensure all team members are committed to achieving specific targeted pathway goals.

Competing interests None declared.

\section{OC-127 ONE THOUSAND LAPAROSCOPIC ROUX-EN-Y GASTRIC BYPASSES: PERIOPERATIVE AND LONG TERM OUTCOMES}

doi:10.1136/gutjnl-2012-302514a.127

J C Tham, * D Pournaras, I Finlay, D Titcomb, J Edmond, D Mahon, R Welbourn. Musgrove Park Hospital, Taunton, UK

Introduction Laparoscopic Roux-en-y gastric bypass (LRYGB) has been established as an effective treatment for obesity and obesity related morbidity. However, safety of LRYGB remains under public scrutiny. Defining operative outcomes is vital to establish its safety and long term efficacy.

Methods One thousand consecutive patients underwent LRYGB as a primary procedure by two surgeons in a single centre from 2004 to 2011. Twenty-six patients had intra-gastric balloon insertion before LRYGB to reduce Body Mass Index (BMI). LRYGB was done using a retrocolic, antegastric linear staple technique with routine closure of internal hernia defects. Patient demographics and complications were collected prospectively in a local database, and after January 2009, in the National Bariatric Surgery Registry. The Obesity Surgery Mortality Risk Score (OS-MRS) was used for risk stratification. Analysis was performed on each 100 patients making up 10 groups sequentially with the first 100 in group 1.
Results Mean age \pm SD was $45.5 \pm 10.4$ with mean BMI of $49.5 \pm 7.7$ and $79.3 \%$ were female. There were 61 (6.1\%) high-risk patients (OS-MRS class C). The follow-up rate up to 6 years was $87.9 \%$. There were four conversions-to-open surgery in the first 400 patients with no conversions thereafter. Mean \% excess weight loss (EWL) pre-operatively as compared to initial weight was $11.6 \% \pm$ 12.5. \% EWL subsequently was: 1 year $67.7 \% \pm 18.9 ; 2$ years: $71.6 \% \pm$ 20.3; 5 years: $57.5 \% \pm 21.8$; 6 years: $61.1 \% \pm 21.4$. Overall, the 30 -day complication rate was $5.5 \%$ with a 30 -day reoperation rate of $2.9 \%$. The 30-day complication rate fell over time; 13 patients had a complication in group 1 compared to 1 patient in group $10(p=0.03)$. Further, there was significant reduction in complications between groups 1 and $2(p<0.001)$ but no difference between each group thereafter. Major complications within 30 days postoperatively were gastrointestinal leak $(0.6 \%)$, gastro-jejunal stenosis $(0.3 \%)$, stenosis at the mesocolic defect $(0.3 \%)$, jejuno-jejunostomy hernia $(0.7 \%)$, mesocolic hernia (1\%) and bleeding $(2.1 \%)$. There was one death $(0.1 \%)$ within the 30 postoperative days (in group 2$)$. The cumulative complication rate at 6 years was $9.1 \%$ with $5.8 \%$ needing re-operation or endoscopic intervention. The mean operative time per group for the first 600 patients was, in minutes, $163 \pm 53.7,129.2 \pm 109.8,100.0 \pm 27.8,104.3 \pm 28.9,92.7 \pm 32.1$ and $93.8 \pm 30.9$, a significant decrease between groups 1 and $2(p=0.003)$, 2 and $3(p=0.007)$, and 4 and $5(p=0.008)$ respectively, but not between 3 and 4 .

Conclusion LRYGB is a safe and efficacious procedure with sustained weight loss at 6 years. The learning curve is about 100 cases but operative time continues to improve up to the 500th case. A high volume centre is associated with low morbidity and mortality.

Competing interests None declared.

\section{OC-128 THE EFFECT OF OBESITY ON THE RADICALITY OF SUBTOTAL OESOPHAGECTOMY FOR OESOPHAGEAL ADENOCARCINOMA}

doi:10.1136/gutjnl-2012-302514a.128

H V Jones, ${ }^{*}$ S Wahed, A Krishnan, J Shenfine, S M Griffin. Northern OesophagoGastric Cancer Unit, Royal Victoria Infirmary, Newcastle Upon Tyne, UK

Introduction Obesity is on the increase in the UK and is a known risk factor for adenocarcinoma of the oesophagus. It is recognised that oesophagectomy in obese patients is more difficult with concerns that radicality of resection is reduced. The aims of this study were to evaluate body mass index (BMI) in patients with oesophageal adenocarcinoma who underwent subtotal oesophagectomy with radical lymphadenectomy and to evaluate the effect of obesity on lymph node (LN) dissection and survival.

Methods All patients who underwent subtotal oesophagectomy for adenocarcinoma between January 2000 and December 2010 were identified from a prospectively maintained database. All other histological types were excluded. Patients were categorised according to BMI using the WHO criteria: underweight (2), normal 
$\left(18.5-24.99 \mathrm{~kg} / \mathrm{m}^{2}\right)$, overweight $\left(25-29.99 \mathrm{~kg} / \mathrm{m}^{2}\right)$ and obese $\left(\geq 30 \mathrm{~kg} / \mathrm{m}^{2}\right)$. Demographics, presence of Barrett's oesophagus or reflux disease, operative time, $\mathrm{R} 0$ resections, complications, LN resection and positivity were analysed. Long-term and disease free survival were calculated using the Kaplan-Meier method.

Results 413 patients were identified. 23 had no BMI recorded and were excluded leaving 390 patients: eight underweight; 117 normal BMI; 172 overweight; 93 obese. BMI significantly increased over time (mean BMI 26.0 in 2000-2001, 27.8 in 2010, $p=0.041$ ). Obese patients were younger compared to normal BMI patients (mean age 60.1 and 64.4 respectively, $p=0.003$ ). The incidence of Barrett's oesophagus and reflux disease were not significantly different between groups. Operating time was significantly longer for obese patients $(p=0.018)$. R0 resections were similar between groups (normal patients $96.4 \%$ and obese $95.5 \%$ ). The mean number of LNs resected (33 for both normal BMI and obese groups) and the LN ratio did not differ significantly between groups. Obese patients had significantly lower disease stages (32.3\% stage 1 obese patients vs $16.2 \%$ stage 1 normal BMI patients, $\mathrm{p}=0.006$ ). Overall survival was longer for obese patients compared with those of normal BMI (81 months vs 55 months, $p=0.004$ ). When matched for stage, this difference did not reach significance $(p=0.236)$. Disease free survival did not differ between groups. The overall complication rate was similar between groups (70.1\% for normal BMI, 66.3\% for obese). Conclusion This is the first study to evaluate BMI in a homogenous group of patients with adenocarcinoma undergoing subtotal oesophagectomy with a standardised radical lymphadenectomy. BMI and obesity among these patients increased with time. The radicality of surgery, in terms of LN yields and R0 resections, did not reduce in the obesity group and this is further supported by equivalent stage-matched long-term survival.

Competing interests None declared.

\section{Free papers AUGIS HPB}

\section{OC-129 METASTATIC PANCREATIC NEUROENDOCRINE TUMOURS: DOES AGGRESSIVE SURGICAL INTERVENTION IMPROVE OUTCOME?}

doi:10.1136/gutjnl-2012-302514a.129

M J White, ${ }^{*}$ L M Edwards, S O Cawich, C Frola, M Abu Hilial, N W Pearce. Department of Surgery, University Hospitals Southampton NHS Trust, Southampton, UK

Introduction Pancreatic neuroendocrine tumours (PNETS) often present late. At diagnosis $65 \%$ of patients have metastases, with median survival of 24 months. ${ }^{1} 2$ The conventional approach is conservative management. Recent evidence has suggested that aggressive treatment leads to better outcomes. ${ }^{3}$ We aim to show that in a cohort of patients with advanced PNETs aggressive resection prolongs survival.

Methods All patients with intra-abdominal neuro-endocrine tumours (NETs) were assessed by the Hepato-pancreatobiliary and NET MDTs from April 2005 to January 2012 were prospectively registered on a database. All patients with PNETs were identified. Demographic, treatment, peri-operative and survival data were analysed using SPSS. Peri-operative morbidity was graded using the Clavien system. Predicted survival was calculated using the Kaplan-Meier method.

Results 239 patients were assessed. 61 patients had PNETs (36 female: 25 males) Median age of 65.55 patients underwent 71 resections. 52 had primary tumours resected and three patients had the primary tumour left in situ. Procedures included: 29 conventional pancreatic resections; 17 extended (including vascular or multi-visceral resections); nine had pancreatic and liver (four synchronous, five sequential) resections; 16 were liver resections alone. 27 patients had 48 additional medical and radiological inter- ventions. Peri-operative mortality was $0 \%$. Overall morbidity $42 \%$. Median blood loss was $700 \mathrm{ml}$. Three operated patients died from progressive disease. Five non-surgical patients died. Overall survival for PNETs was $92 \%$ at median follow-up of 3 years. Predicted 5 year survival rate was $72 \%$ in operated patients.

Conclusion An aggressive multi-modal approach with resection of advanced PNETs leads to excellent long term survival with acceptable morbidity in patients.

Competing interests None declared.

\section{REFERENCES}

1. Yao JC, et al. Everolimus for advanced pancreatic neuroendocrine tumours. $N$ Eng/ J Med 2011;364:514-23.

2. Yao JC, et al. One hundred years after "carcinoid": epidemiology of and prognostic factors for neuroendocrine tumours in 35,825 cases in the United States. J Clin Oncol 2008;26:3063-72.

3. Ong SL, et al. A fuller understanding of pancreatic neuroendocrine tumours combined with aggressive managemment improves outcome. Pancreatology 2009;9:583-600.

\section{OC-130 EMERGENCY CHOLECYSTECTOMY; AN ECONOMIC EVALUATION OF PRACTICE AT A REGIONAL HEPATOBILIARY CENTRE}

doi:10.1136/gutjnl-2012-302514a.130

${ }^{1} \mathrm{~N}$ Misra, ${ }^{*} \mathrm{~V}$ Kaliyaperumal, ${ }^{2} \mathrm{~N}$ Grimes, ${ }^{2} \mathrm{E}$ McChesney, ${ }^{2} \mathrm{R}$ Jones, ${ }^{1} \mathrm{D}$ Dunne, ${ }^{1} \mathrm{G}$ Poston, ${ }^{1} \mathrm{~S}$ Fenwick, ${ }^{1} \mathrm{H}$ Malik. ${ }^{1}$ North Western Hepatobiliary Centre, University Hospital Aintree, Liverpool, UK; ${ }^{2}$ University of Liverpool, Liverpool, UK

Introduction The debate as to how to best manage patients presenting acutely with complications of gallstones continueswhether to consider early emergency surgery or not. Perceptions of increased risk and greatly increased cost still persist about the early approach. We report on our experience from a regional hepatobiliary centre.

Methods A retrospective clinical study was conducted of all patients admitted with acute biliary symptoms, and who underwent cholecystectomy between January 2008 and August 2011. Costing data were calculated for each patient on an individual basis, including all theatre consumables, drugs and calculated cost for length of stay. A decision tree analysis economic model was created, using input data derived from the clinical study as well as the individual patient level costs, and uncertainty in this model tested with probabilistic sensitivity analyses. Categorical data were analysed using the $\chi^{2}$ test.

Results Of the 1888 patients who had a cholecystectomy during this period, 89 had an emergency or early laparoscopic cholecysytectomy (eLC) and 310 patients presented acutely with biliary disease and then went on to have a delayed cholecystectomy (dLC). Overall median length of stay (LoS) for the eLC group was 6 days, and for the delayed group was 7 days $(p=N S)$, including the primary admission for medical treatment. The emergency readmission rate for all patients on the waiting list was $13 \%$ with a median stay of 4.5 days. Post-operative readmission rates were equivalent for both eLC $(8 \%)$ and $\operatorname{dLC}(9 \%)-p=N S$. Mean operating time was longer in the eLC group than the dLC group $-120 \mathrm{~min}$ vs $60 \mathrm{~min}(\mathrm{p}<0.05)$. Post operative ERCP rates were $3 \%$ for the eLC group and $0 \%$ for the dLC group $(p=N S)$, post operative fluid collections requiring intervention were $6 \%$ for the eLC group as opposed to $0 \%$ for the $\mathrm{dLC}$ group ( $p=N S$ ). The baseline cost difference between the eLC and dLC groups was around $£ 150$ more expensive for the eLC group. After complications and readmission costs were calculated and inputted into the decision tree analysis, this difference decreased to a cost of $£ 52$ - more expensive for the eLC pathway.

Conclusion Early cholecystectomy on the index admission appears to be safe, with overall hospital stay slightly shorter. The difference in costs between the early and delayed pathway was essentially cost equivalent. But with NHS tariff (around $£ 3650$ for eLC and $£ 2900$ 